\title{
Development of Distance Learning Concept with Graphic Machines Working Process Visualization
}

\author{
Zeljko Zeljkovic ${ }^{1, *}$, Dragoljub Novakovic ${ }^{1}$, Sandra Dedijer $^{1}$, Nemanja Kasikovic ${ }^{1}$, and \\ Stefan Djurdjevic ${ }^{1}$ \\ ${ }^{1}$ University of Novi Sad, Faculty of Technical Sciences, Department of Graphic Engineering and \\ Design, Trg Dositeja Obradovica 6, Novi Sad, Serbia
}

\begin{abstract}
The paper presents the distance learning concept development by visualizing the work process of graphics machines. The basic goal is realized through the application development that includes machines simulations and their elements. A large number of simulations and explanations of the work process are included in the knowledge base. The concept is expanded to learning how to program machines so that the future operator can master significant knowledge that will enable him to engage in the production process quickly. The paper presents concrete types of machines for different printing techniques and machines for making packaging prototypes. For the realization of objects visualizations, modern software tools were used, which finally provided good quality.
\end{abstract}

\section{Introduction}

Information revolution brought improvements to every sector of our lives, and education represents one of the most important sectors which can benefit immensely from these improvements. The most important improvement brought by the information revolution is accessibility which allows easier and faster access to information. E-learning refers to any kinds of the use of electronic devices for learning the purpose, including the delivery of content via electronic media such as computer network, audio, video, satellite broadcast, interactive TV, and so on [1]. As learning has become more individualized, learner-centred, situated, collaborative, and ubiquitous; e-learning technology has become more personalized, user-centred, networked, ubiquitous, and durable [2]. Distance education is defined as "teaching through the use of telecommunications technologies to transmit and receive various materials through voice, video and data" [3]. In the same token, Leidner and Jarvenpaa define distance learning as "the transmission of a course from one location to another" [4]. These definitions provide an analogy to distance learning in the field of information technology or online software training. Online software training can be the transmission of instructional IT programming or contents to geographically dispersed individuals or groups. [5] The paper presents research focused on developing the software knowledge base in the form of interactive application, which would be included in the distance learning project, in particular, the application visualizes a concrete type of

\footnotetext{
* Corresponding author: zelikoz@uns.ac.rs
} 
machines for different printing techniques and machines for making packaging prototypes. The application includes a visualization of the main parts of the machine, the interaction between them and the additional descriptive text documents. The research aims to make the online environment for the future operator to master significant knowledge that will enable him to engage in the production process quickly.

\subsection{Offset printing}

Offset printing is a commonly used printing technique. In the offset printing process, printing and non-printing elements are in apparently the same place on the printing plate. The printing elements have the characteristic that they accept, and the non-printing elements refuse the inks. Offset printing is an indirect printing process. The inked image is transferred (or "offset") from a plate to a rubber blanket, then to the printing surface. Offset printing can produce a full range of print media in high quality from a single prospect to a high-quality catalogue. [6]

\subsection{Pad printing}

The pad printing, also called tampography, works on the principle of gravure printing, which means that the printing elements on the printing form are at a lower level compared to the non-printing elements. This printing technique is an indirect printing process that involves an image being transferred from the printing form via a silicone pad onto a substrate. This allows printing on a variety of surfaces, such as flat, cylindrical, spherical, compound angles, textures, concave, or convex surfaces [7].

\subsection{Cutting machine}

Flatbed cutters are computer-controlled large format machines for the precise $\mathrm{CAD} / \mathrm{CAM}$ processing of all non-metallic substances, rigid or flexible surfaces. It is certain that computer-aided design (CAD) has revolutionized modern engineering. In the world of engineering, CAD is extremely important and widely used for designing and developing packaging products. CAD increases the productivity of engineers/designers, improves design quality. The main goal of the packaging prototype design is the ability to test the physical characteristics and behavior of the packaging. Therefore, it is of the utmost importance that the prototype is as close as possible to the characteristics of the final packaging. Today CAD / CAM systems are used primarily for making prototype cardboard packages, where they are cut out by CAD drawings [8].

\section{Distance Learning Concept Development}

After we choose the machine for creating distance learning concept, we research information such as blueprints (scatches of the graphic machine) and how the machine works. After that, it is necessary to create $3 \mathrm{~d}$ models of the graphic machine, and apply textures and materials on it. Next step is creating animations of the graphic machine, animating objects, textures and materials. In the post-production phase, $3 \mathrm{~d}$ animations are rendered and composed in video clips. In final steps using video clips and text files from research step we create a distance learning environment and make it interactive to finalize the distance learning application (Figure 1). 


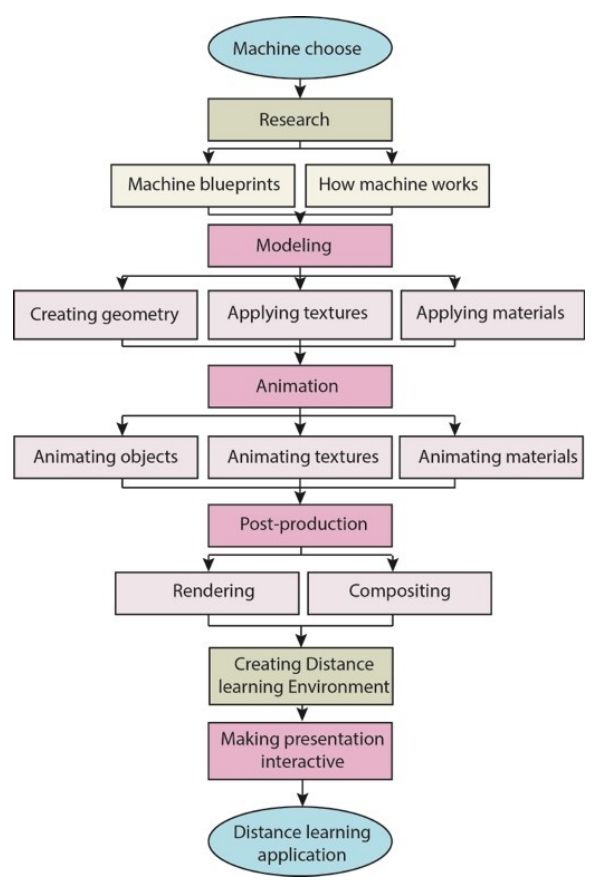

Fig. 1. Entire process flowchart.

\subsection{Modeling}

Modeling is the process of creating three-dimensional models in a virtual environment, where in this concept the polygonal modeling with the use of modifiers is most often used. [9] Modeling usually starts from geometric primitives, such as a plate or cube object, whose geometry is then modified. The most common manipulations rely on adding geometry or spatial displacement of existing geometry. For a more accurate and simplified modeling process, modifiers that have many applications, such as mirror modifiers, are applied (Figure 2).

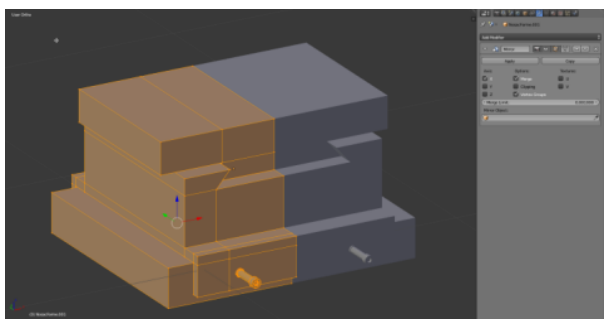

Fig. 2. 3D model modified with a mirror modifier.

The modeling order is generally relative. However, it is necessary to consider the purpose of the objects being modelled, especially when models will be animated. In this case, this means that the objects that are animated are modelled first, with additional testing of the animation process. 

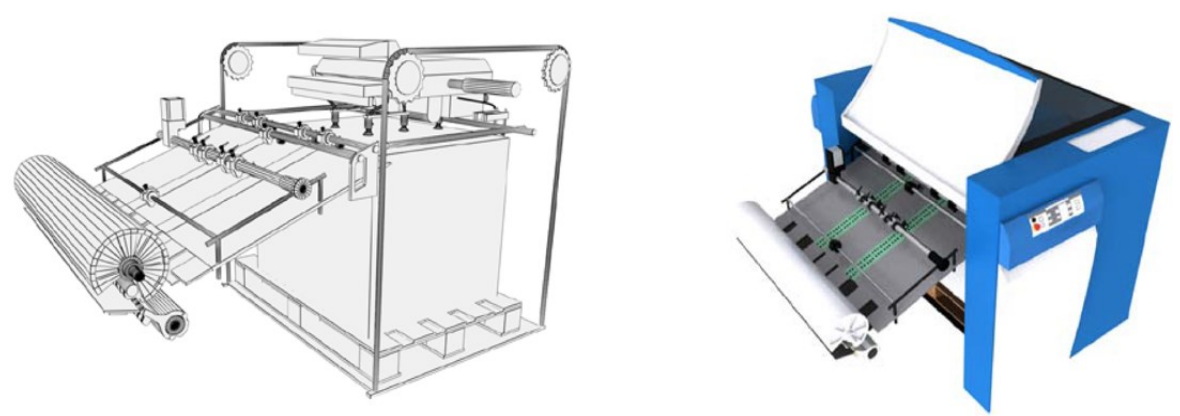

Fig. 3. 3D model of the machine part, wireframe left and render right.

Figure 3 shows the detail of the offset printing machine wireframe model. As a result of the modeling, the complete scene with objects that represent the elements of the machine contains 1757 objects with a total of approximately 270000 polygons.

\subsection{Applying textures and materials}

The complexity of this process depends on the project itself, and in the case of visualization, a clearer picture is often required. That means it is often necessary to use materials with simple surfaces and it is not necessary to use textures for all objects. The process of applying materials relies on changing the basic properties of materials such as color, surface diffusion, reflectivity, etc. Materials are based on the appearance of realworld material.

Besides simple surfaces, it is also necessary to use textures for certain objects, such as parts of machines with labels. First, we created a UV map of the model, that indicates the textures location on the 2D surface of the model, and later we added an image to that surface. The texture we created in the vector software Adobe Illustrator, and we exported it as a raster image to be readable as UV texture.

\subsection{Animation}

The animation process in 3D software is mainly based on the use of keyframes that represent the points in the timeline in which the object changes specific properties. The animation is created based on the calculation that software does on the timeline between keyframes (Figure 4). For animation purposes, several types of animations are used, where the simplest type is directly animating the position or the rotation of the object. Also, we use the animation of the object along a closed path, where the object moves based on the position on the path.

The last type of animation used is material property control, where certain values of material properties and textures are animated to simulate the print process for example. These parts are animated with a simple texture replacement or changing the state of object reflectivity.

\subsection{Post-production}

Post-production is the final stage of production, and the reliance is mainly made up of the rendering and compositing process. Rendering represents the process of virtual recording of digital scenes and models on the scenes, and the composition is the process of adding elements into a particular video clip. In this case, the largest video content elements are 
rendered without additional compositing, while the composite is used for the cleaning process to shorten the rendering process.

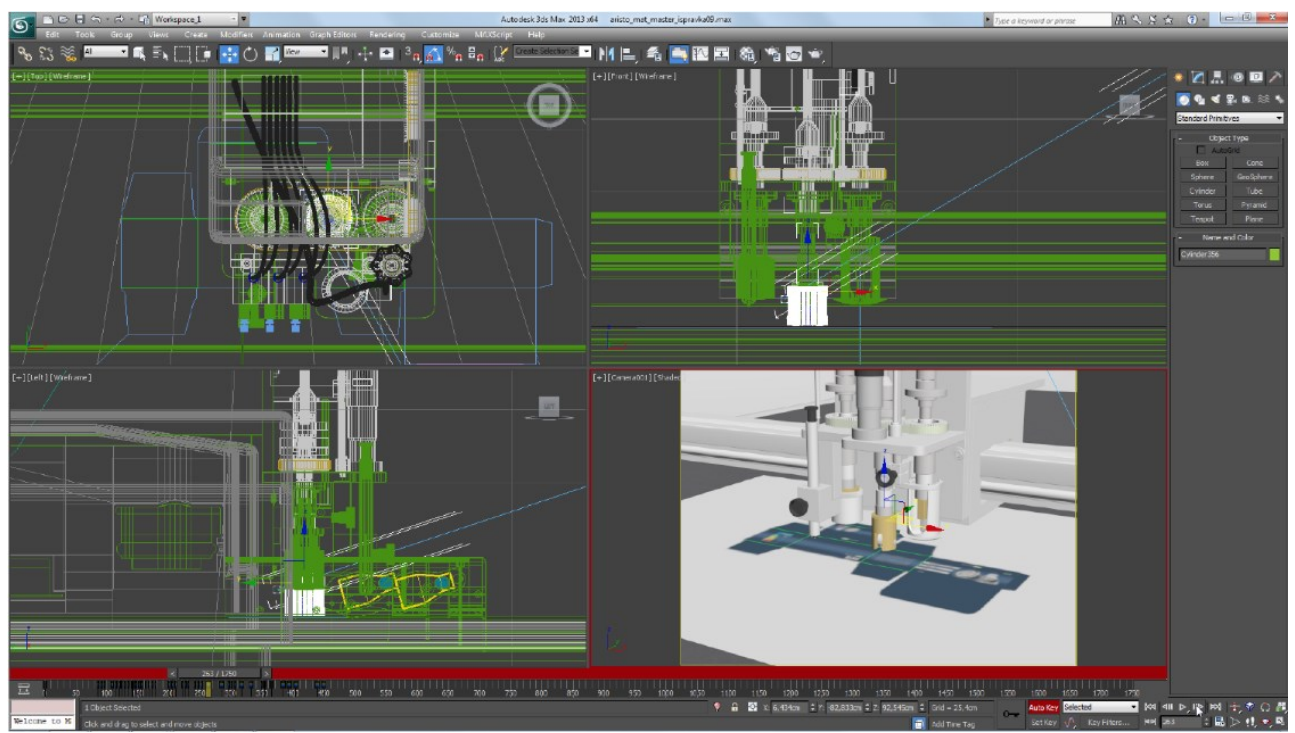

Fig. 4. Cutting machine animation process timeline and keyframes.

\subsection{Interactive presentation}

In the previous step, the process of making all necessary video sequences, pictures and texts have been done, and then it is necessary to organize all information effectively in order to provide the distance learning environment.

The main characteristic that was necessary to achieve through the development of this environment is interactivity. Interactivity plays a very large role in the way and the speed of the users learning process.

Adobe Flash refers to a multimedia software used to create materials for the Adobe Engagement Platform, such as web applications, games, and movies. It supports vector and raster graphics, a script programming language called ActionScript and two-way streaming audio and video signals.

More specifically, Adobe Flash is an integrated development environment (IDE), while Flash Player is a virtual machine that is used to play Flash files. The term Flash can be used for both the program environment and the player as well for files that come from the program. [7]

Flash application is the result of the final form of the distance learning concept. All mounted video sequences exported from the Adobe Premier application as AVI files are imported within the Flash timeline and then stopped and programmed in animation frames to allow interaction.

In these frames, it is possible to click on individual parts of machines and read more detailed information about the parts of the machine itself. The information is made up of images, video sequences and extra text that is loaded from an external text document. Figure 5 shows the main view of the application. 


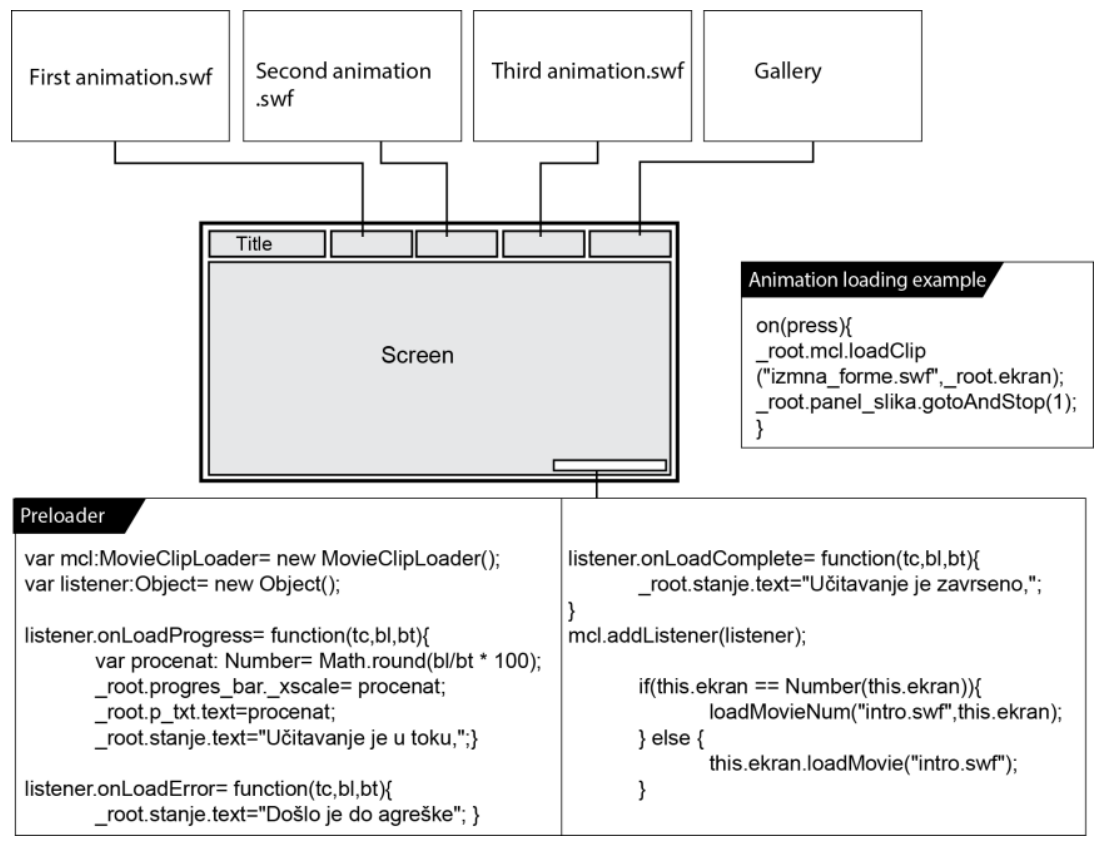

Fig. 5. The main view of the Distance learning application.

An additional text describing the parts of the machine is loaded from external text documents that can be modified later without the modifying in Flash application.

In addition to the text that is loaded when we click on certain parts of the machine, images and specific video sequences recorded on the real machine are loaded to make the user familiar with the operation of the machines. Figure 6 shows the distance learning application user interfaces.

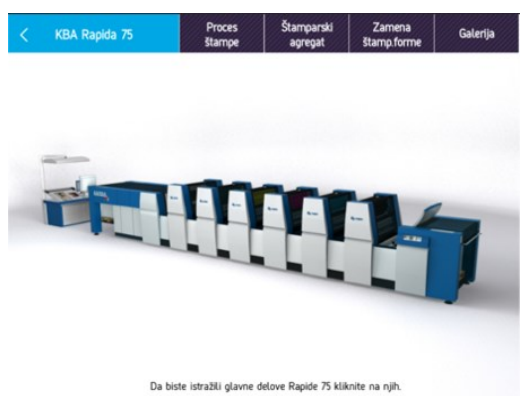

a)

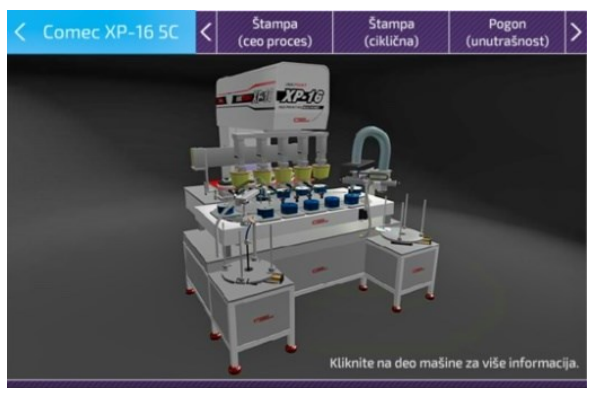

b)

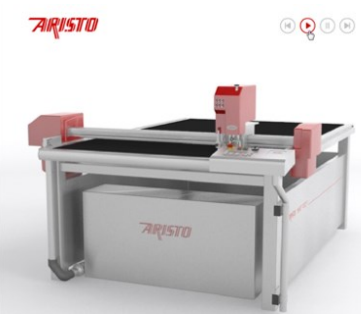

c)

Fig. 6. Distance learning application user interfaces a) Offset printing machine, b) Pad printing machine, c) Cutting machine. 


\section{Conclusion}

The result of this work is working interactive application for the distance learning concept. The need to effectively present the generated information about offset printing, pad printing and cutting machine is established. Also, the main models of the machines have been made, but modeling can be extended with more details such as the drive and pneumatic system of offset machine.

The final application is exported as an executable file that runs without the need to install additional programs. The application can also be integrated easily in the modern HTML site so it can be accessible to a large number of users.

Acknowledgement: This work was supported by the Serbian Ministry of Science and Technological Development, Grant No.: 35027 "The development of software model for improvement of knowledge and production in graphic arts industry".

\section{References}

1. D. Shee, \& Y. H. Wang, Comput Educ., 50, 894-905 (2008)

2. L. F. Motiwalla, Comput Educ., 49(3), 581-596 (2007)

3. A. Bielefield, L. Cheeseman, Technology and copyright law. New York: Neal-Schuman Publishers Inc, 141 (1997)

4. D. E. Leidner, S. L. Jarvenpaa, MIS Quarterly, 19, 265-291 (1995)

5. M. G. Moore, Handbook of Distance Education (2013)

6. H. Kipphan, Handbook of Print Media (2001)

7. Teca-Print, The pad printing overview: pad printing, printing pads, plates, pad printing inks, adhesion und antistatic, Available at: https://teca-print.com/pdf_eng/ Tampondruck verfahren/The_pad_printing_process_706-000-465.pdf (Accessed: 23rd February 2019)

8. U.S. Congress, Office of Technology Assessment, Computerized manufacturing automation. DIANE Publishing. 48 (1984)

9. K. L. Murdock, Autodesk 3Ds Max 2014 (2014) 